\title{
Stable expression of temperature-sensitive p53: A suitable model to study wild-type p53 function in pancreatic carcinoma cells
}

\author{
DIETER NUEVEMANN, MATTHIAS CHRISTGEN, HENDRIK UNGEFROREN and HOLGER KALTHOFF \\ Department of General and Thoracic Surgery, Research Unit Molecular Oncology, University Hospital \\ Schleswig-Holstein, Campus Kiel, Arnold-Heller-Strasse 7, D-24105 Kiel, Germany
}

Received January 24, 2006; Accepted March 7, 2006

\begin{abstract}
Pancreatic adenocarcinoma is an extremely aggressive malignancy with a dismal prognosis. Inactivation of the p53 tumor-suppressor gene occurs in approximately $50 \%$ of primary tumors and is thought to account for a failure of the tumor cells to undergo growth arrest and apoptosis in response to chemotherapy. Hence, it is of interest to study the consequences of the restoration of wild-type (wt) p53 function in pancreatic carcinoma cells. Therefore, we retrovirally transduced temperature-sensitive (ts) human p53 into the p53-null pancreatic carcinoma cell line AsPC-1. ts p53 has a mutant phenotype at $37.5^{\circ} \mathrm{C}$, and a wt conformation at $32.5^{\circ} \mathrm{C}$. Stable expression of $\mathrm{p} 53$ in wt conformation caused upregulation of the $\mathrm{p} 53$ responsive gene $p 21^{\text {Wafl/Cipl }}$, and $\mathrm{G}_{1}$ growth arrest, but failed to induce Bax expression or apoptosis. In addition, we examined the effect of wt p53 expression on DNA damaging treatment. Interestingly, the doxorubicin- and radiation-induced $\mathrm{S}-/ \mathrm{G}_{2}$-phase arrests were suppressed by $\mathrm{p} 53$ in wt conformation. These results demonstrate that the ts $53 / \mathrm{AsPC}-1$ model is suitable to investigate the effect of wt $\mathrm{p} 53$ restoration in pancreatic carcinoma cells.
\end{abstract}

\section{Introduction}

Adenocarcinoma of the pancreas is an extremely aggressive malignancy with a dismal prognosis (1). Development of pancreatic cancer is a multi-stage process resulting from the accumulation of somatic DNA mutations in normal cells. The major genetic alterations affect the K-ras oncogene, which is frequently activated by somatic mutations, and the tumorsuppressor genes, $p 53, p 16$ and DPC4, which are inactivated in pancreatic cancer. Inactivation of the p53 tumor-suppressor gene is observed in approximately $50 \%$ of primary pancreatic carcinomas, and in almost all of these cases this inactivation

Correspondence to: Dr Hendrik Ungefroren, Department of General and Thoracic Surgery, Research Unit Molecular Oncology, University Hospital Schleswig-Holstein, Campus Kiel, Arnold-Heller-Strasse 7, D-24105 Kiel, Germany

E-mail: hungefroren@chirurgie-sh.de

Key words: AsPC-1, tsp53, p21waf1, bax, cell cycle occurs by loss of heterozygosity (LOH) associated with an intragenic mutation of the remaining allele (2). However, p53 gene mutations are much more overrepresented in established pancreatic carcinoma cell lines. Moore et al recently found 21 out of 22 cell lines to harbour $p 53$ gene mutations (3). p53 responsive genes, such as $p 21^{\text {Wafl/Cipl }}$ and Bax, induce cell cycle arrest or apoptosis (4). Therefore, the development of chemoresistance and tumor progression is largely attributed to the disruption of the normal p53 function. However, it is well documented that pancreatic carcinomas with wild-type (wt) p53, are extraordinarily aggressive malignancies and exhibit considerable chemoresistence (5). The lack of cell lines with wt p53 requires ectopic expression of wt p53 to study p53 functions in pancreatic carcinoma cell lines. Unfortunately, as mentioned above, most pancreatic carcinoma cell lines synthesize mutated p53 proteins, which potentially suppress the function of ectopically expressed wt $\mathrm{p} 53$ protein by their dominant negative activity (6). Therefore, only a p53-null pancreatic carcinoma cell line would represent a suitable model to investigate the consequences of ectopic wt p53 expression. The pancreatic carcinoma cell line AsPC-1 fulfils this requirement. AsPC-1 cells harbour two mutated p53 alleles, and completely lack p53 mRNA transcripts (7). Previously, Kimura et al reintroduced wt p53 in AsPC-1 cells by retroviral infection (8). Due to the permanent p53-mediated growth inhibition, this approach required the tedious expansion of cell clones in long-term cultures. This step might have led to the selective expansion of subclones not representative of the original cell line (9). To avoid long-term culturing of cells permanently expressing wt p53 we employed a temperaturesensitive (ts) human p53 gene (10). ts p53 has a mutant phenotype at $37.5^{\circ} \mathrm{C}$ (restrictive temperature), and a wt conformation at $32.5^{\circ} \mathrm{C}$ (permissive temperature). In this study we show that AsPC- 1 cells retrovirally transduced with ts p53 are suitable to investigate p53 functions in clones isolated without selective pressure of permanent wt p53 expression. By using this novel cellular model we analysed the impact of wt p53 on cell cycle regulation and apoptosis of AsPC-1 cells following doxorubicin treatment and $\gamma$-irradiation.

\section{Materials and methods}

Cell line and culture conditions. The human pancreatic cancer cell line AsPC-1 was obtained from the American Type Culture Collection (Rockville, MD, USA) and routinely maintained in RMPI-1640 supplemented with 10\% FCS, 2 mM 
glutamine and $1 \mathrm{mM}$ sodium pyruvate (Life Technologies, Inc., Eggenstein, Germany) without penicillin or streptomycin (11). Retrovirally transduced cells expressing ts 553 received $700 \mu \mathrm{g} /$ $\mathrm{ml}$ geneticin (Sigma, Deisenhofen, Germany) in addition. All cells were kept in a water-saturated atmosphere containing $5 \% \mathrm{CO}_{2}$ at $37.5^{\circ} \mathrm{C}$, and pre-warmed medium was used in all medium changes. For treatment with DNA damaging agents, the cells were seeded at $70 \%$ confluence in 6-well plates and were allowed to adhere overnight at $37.5^{\circ} \mathrm{C}$. Cells were then incubated for an additional $8 \mathrm{~h}$ at either 37.5 or $32.5^{\circ} \mathrm{C}$ and subsequently exposed to $0.1 \mu \mathrm{g} / \mathrm{ml}$ doxorubicin (Pfizer, Switzerland), 8 Gy of $\gamma$-irradiation (Gammacell 40, Anatomic Energy of Canada Ltd.) or remained untreated for $24 \mathrm{~h}$.

Generation of retroviral vectors and retroviral infection. The cDNA for the human temperature-sensitive p53 mutant, p53Val-138 (amino acid 138, Alanine is changed into Valin), in pCMVnb was kindly provided by Dr N. Tsuchida (Tokyo Medical and Dental University, Japan) (10). The ts p53 cDNA was released from pCMVnb with BamHI followed by polishment of both ends with Klenow fragment and was subcloned in sense orientation into the PmeI site of the retroviral vector TJBa5bMoLink-neo (TJM). This construct was co-transfected into human embryonic kidney $293 \mathrm{~T}$ producer cells, along with retroviral packaging vectors, as described previously (12). Conditioned medium from 293T cells containing retroviral particles was used to transduce AsPC-1 cells in an overnight incubation.

Immunological reagents. The mouse monoclonal antibody (mAb) NCL-53-DO1 against human p53 was purchased from Novocastra Laboratories (Newcastle, UK). NCL-53-DO1 likewise detects p53, and ts p53 in wild-type and mutant conformation. The mouse mAb against human $\mathrm{p} 21^{\text {Waf1/Cip1 }}$ (clone 70) was purchased from BD Transduction Laboratories (Hamburg, Germany) and the rabbit polyclonal antibody against human Bax was purchased from Pharmingen (Hamburg, Germany). Anti- $\beta$-actin (clone AC-15) antibody was obtained from Sigma, peroxidase-conjugated sheep anti-mouse $\operatorname{IgG}$ was obtained from Amersham (Braunschweig, Germany), biotin-conjugated goat anti-mouse IgG and streptavidin-alkaline phosphatase conjugate were obtained from Tropix, Applied Biosystems (Massachusetts, USA).

Western blotting. For Western blotting, $30 \mu \mathrm{g}$ of total cellular protein (measured with BCA Protein Assay Reagent, Pierce Chemical Co., IL, USA, according to the manufacturer's instructions) were separated by $12 \%$ SDS-PAGE and transferred to a polyvinylidene difluoride membrane (PVDF) (Immobilon-P; Millipore, Eschborn, Germany). Membranes were blocked with PBS containing 5\% non-fat dry milk overnight at $4^{\circ} \mathrm{C}$, washed several times with PBS containing $0.2 \%$ Tween-20 (PBS/Tween) and then incubated with the primary antibody. After washing with PBS/Tween, blots were incubated with appropriate secondary antibody. Peroxidase-conjugated secondary antibodies against primary anti-p53 mAb and p $21^{\text {Waf } 1 / \text { Cip } 1} \mathrm{mAb}$ were developed with the chemiluminescent detection kit ECL (Amersham). The biotin-conjugated secondary antibody against primary anti-Bax antibody with its corresponding streptavidin-alkaline phosphatase conjugate

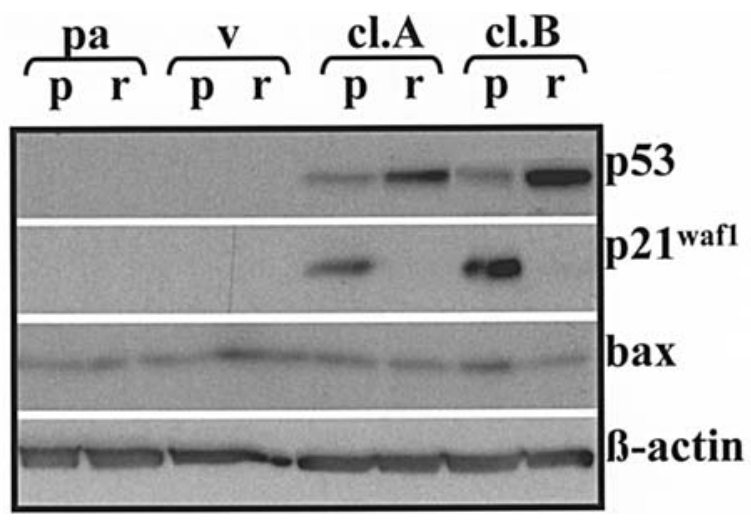

Figure 1. Western blot analysis of $\mathrm{p} 53, \mathrm{p} 21^{\text {Waf } 1 / \mathrm{Cip} 1}$ and Bax protein expression in AsPC-1-pa, AsPC-1-v, AsPC-1-cl.A and -cl.B cells $24 \mathrm{~h}$ after temperature shift to permissive (p) or restrictive (r) temperature. The temperature shift does not affect the expression of $\beta$-actin. The detection of $\beta$-actin demonstrates equal protein loading.

was developed with the chemiluminescent detection kit CDP-Star (Tropix, Applied Biosystems) following the manufacturer's protocol.

Cell cycle analysis. For fluorescence-activated flow cytometry, $4 \times 10^{5}$ cells were obtained from monolayer culture by trypsination at 37.5 or $32.5^{\circ} \mathrm{C}$. Following centrifugation $(10 \mathrm{~min}$ at $600 \mathrm{x} \mathrm{g}$ ), the cell pellet was resuspended in PBS containing $5 \mathrm{mM}$ EDTA and fixed in $50 \%$ ethanol for $30 \mathrm{~min}$ at RT. Cells were subjected to RNAse A treatment by $0.5 \mathrm{ml}$ PBS/EDTA containing $40 \mu \mathrm{g} / \mathrm{ml}$ RNAse A (Boehringer, Mannheim, Germany) for $30 \mathrm{~min}$ at RT. Finally, cells were resuspended in $0.5 \mathrm{ml}$ PBS/EDTA containing $50 \mu \mathrm{g} / \mathrm{ml}$ propidium iodide and incubated for $30 \mathrm{~min}$ at $4{ }^{\circ} \mathrm{C}$ in the dark. DNA content was analysed by flow cytometry using a FACScan (Becton-Dickinson). A total of 10000 cells were counted, cellular debris excluded, and cell cycle distribution was assessed using 'CellQuest' software.

\section{Results}

The ts $p 53$ induces $p 21^{\text {Wafl/Cipl }}$ following temperature shift to permissive temperature. AsPC-1 cells harbour mutated p53 alleles and lack p53 mRNA transcripts (7). The absence of endogenous p53 protein was confirmed by Western blotting of total cellular protein from parental AsPC-1 cells (AsPC-1-pa) (Fig. 1, top). To investigate the consequences of wt p53 restoration in AsPC-1 cells, we introduced the cDNA of ts p53 into AsPC-1 cells by retroviral transduction, followed by geneticin selection of individual clones. Further experiments were carried out with two clones (AsPC-1-cl.A and -cl.B), which exhibited strong expression of ts p53 (Fig. 1, top). AsPC-1-pa cells and AsPC-1 cells transduced with an empty vector (AsPC-1-v) were employed as controls. The level of ts p53 expression in AsPC-1-cl.A and -cl.B was significantly higher at the restrictive temperature and decreased following a shift to the permissive temperature (Fig. 1, top). Expression of ts p53 in its wt conformation (at the permissive temperature) but not in its mutant conformation (at the restrictive temperature) resulted in an upregulation of the p53 responsive 


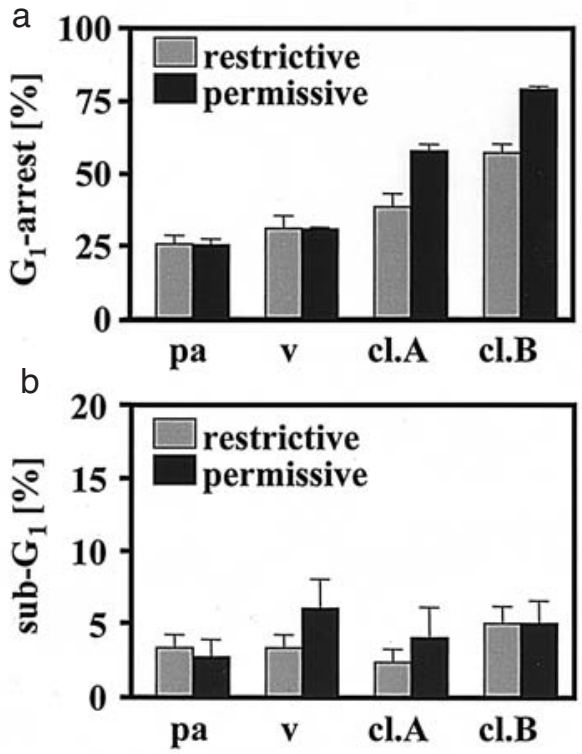

Figure 2. (a) Flow cytometric analysis of cell cycle distribution after temperature shift to restrictive (r) or permissive (p) temperature for $24 \mathrm{~h}$. The bar chart presents means \pm SEM of $\mathrm{G}_{1}$-arrested cells of three independent experiments. (b) Flow cytometric analysis of cell cycle distribution after temperature shift to restrictive (r) or permissive (p) temperature for $24 \mathrm{~h}$. The bar chart presents means \pm SEM of sub- $\mathrm{G}_{1}$ cells of three independent experiments.

gene $p 21^{\text {Wafl/Cipl }}$, indicative of proper ts p53 function (13) (Fig. 1). In contrast, the expression of Bax, a pro-apoptotic gene that has been reported to be transcriptionally induced by p53 (14), remained unaltered following a shift to the permissive temperature (Fig. 1).

The ts p53 induces G1-arrest at the permissive temperature. p53-dependent expression of $\mathrm{p} 21^{\text {Waf1/Cip1 }}$ results in $\mathrm{G}_{1}$-arrest (13). Consistently, at the permissive temperature, AsPC-1-cl.A and -cl.B cells showed an increase in the $\mathrm{G}_{1}$-cell-fraction when compared to AsPC-1-pa and AsPC-1-v cells (Fig. 2a). In addition, p53 mediates apoptosis by transcriptional activation of a variety of pro-apoptotic genes, such as Bax (4). However, neither AsPC-1-cl.A/-cl.B nor AsPC-1-pa/-v displayed a statistically significant increase in the sub- $\mathrm{G}_{1}$ fraction, indicative of apoptosis, at the permissive temperature (Fig. 2b). Nevertheless, the observed $\mathrm{G}_{1}$-arrest at the permissive temperature clearly demonstrates the functional activity of ts p53 in AsPC1-cl.A and -cl.B cells (Fig. 2a).

The ts 553 suppresses DNA damage-induced $S$-/ $G_{2}$-arrests at the permissive temperature. As part of the stress response program wt p53 is upregulated in response to DNA damaging agents at the transcriptional and posttranscriptional level. However, the ability of p53 to induce individual target genes, such as $p 53 A I P 1$, requires distinct posttranslational modifications (15). DNA damaging drugs and $\gamma$-irradiation both stimulate the posttranslational modification of p53, and thus, alter the pattern of expressed p53 responsive genes $(15,16)$. Hence, we exposed the various AsPC-1 derivatives to doxorubicin or $\gamma$-radiation following the temperature shift. A slightly pronounced expression of $p 21^{\text {Wafl/Cipl }}$ was observed following

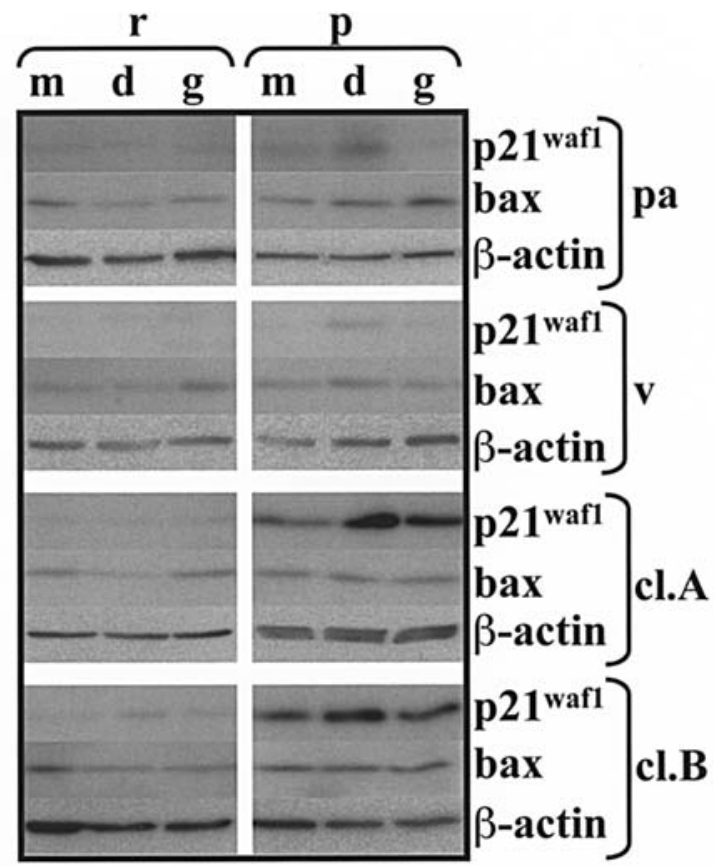

Figure 3. Western blot analysis of $\mathrm{p} 21^{\text {Waf1/Cip1 }}$ and Bax protein expression after temperature shift and treatment with doxorubicin (d), $\gamma$-radiation (g) or medium (m). Cells were shifted to restrictive (r) or permissive (p) temperature for $24 \mathrm{~h}$, followed by DNA damaging treatment for $24 \mathrm{~h}$ at restrictive or permissive temperature, before lysates were prepared.

doxorubicin treatment of AsPC-1-cl.A and -cl.B at permissive temperature (Fig. 3). Again, expression of ts p53 at the permissive temperature and exposure to doxorubicin or $\gamma$-radiation both failed to cooperatively upregulate Bax expression in AsPC-1-cl.A and -cl.B clones (Fig. 3). Remarkably, regarding the cell cycle distribution, AsPC-1-cl.A and -cl.B exhibited an $\mathrm{S}$-arrest following doxorubicin treatment at the restrictive temperature, which was absent at the permissive temperature. In contrast, AsPC-1-cl.A and -cl.B expressing ts p53 at wt conformation at permissive temperature were all $\mathrm{G}_{1}$-arrested and did not respond to doxorubicin with respect to changes in the cell cycle distribution (Fig. 4a and b). In particular, doxorubicin-treated AsPC-1-cl.A and -cl.B did not show a higher number of apoptotic cells at the permissive temperature (Fig. 4a and b). AsPC-1-cl.A and -cl.B exhibited a $\mathrm{G}_{2}$-arrest following $\gamma$-radiation at the restrictive temperature, which was absent at the permissive temperature (Fig. $4 \mathrm{a}$ and $\mathrm{b}$ ). At the permissive temperature, AsPC-1-cl.A and -cl.B were $\mathrm{G}_{1}$-arrested and did not respond to $\gamma$-radiation in terms of changes in the cell cycle distribution (Fig. 4a and b).

The AsPC-1-pa and AsPC-1-v cells exhibited a $\mathrm{G}_{2}$-arrest following doxorubicin treatment at the restrictive temperature, which was also observed, albeit to a lesser degree, at permissive temperature (Fig. 4c and d). In contrast, AsPC-1-cl.A and -cl.B exhibited an S-arrest following doxorubicin treatment at the restrictive temperature. This discrepancy might be explained by a particular, yet aberrant, function of ts p53 in the mutant conformation, which has also been demonstrated for mutant p53. The AsPC-1-pa and AsPC-1-v cells exhibited a $\mathrm{G}_{2}$-arrest following $\gamma$-radiation treatment at the restrictive temperature, which was, to a lesser degree, also observed at the permissive temperature (Fig. $4 \mathrm{c}$ and d). A dominant $\mathrm{G}_{1}$-arrest 
a)
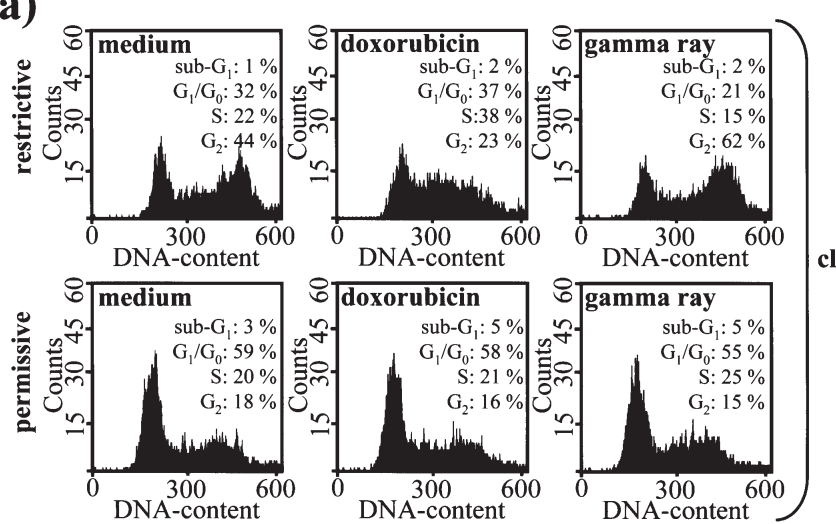

b)
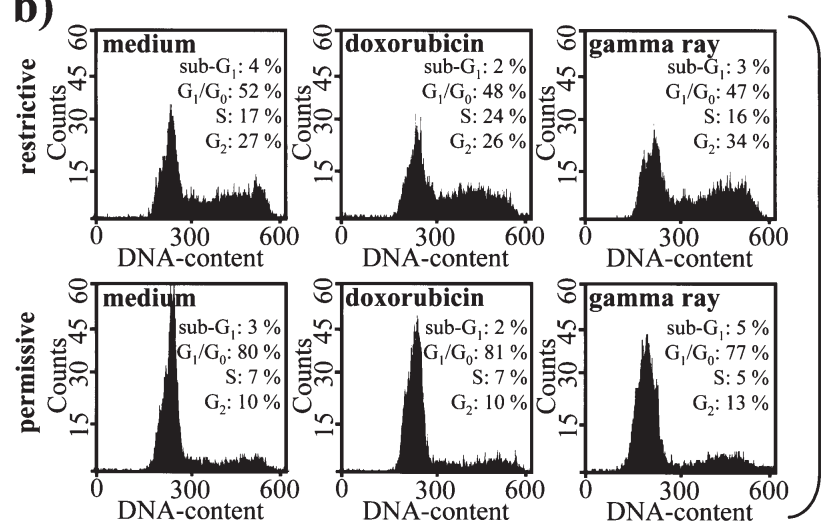

c)
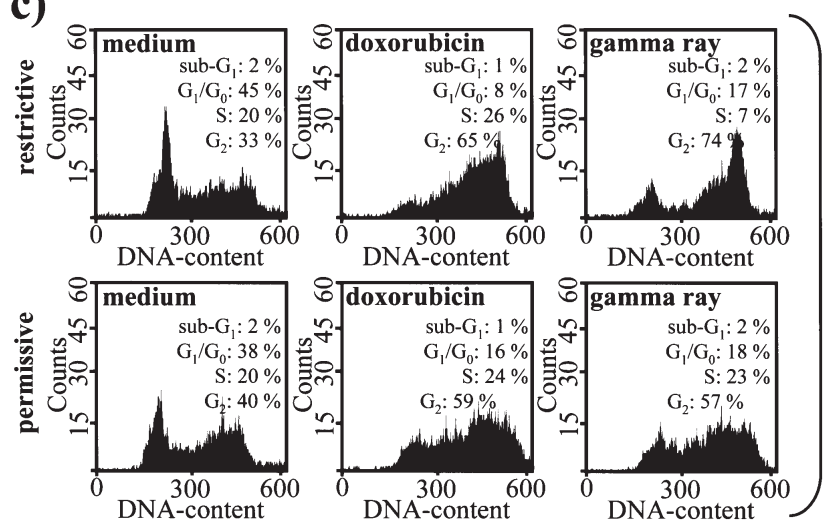

d)
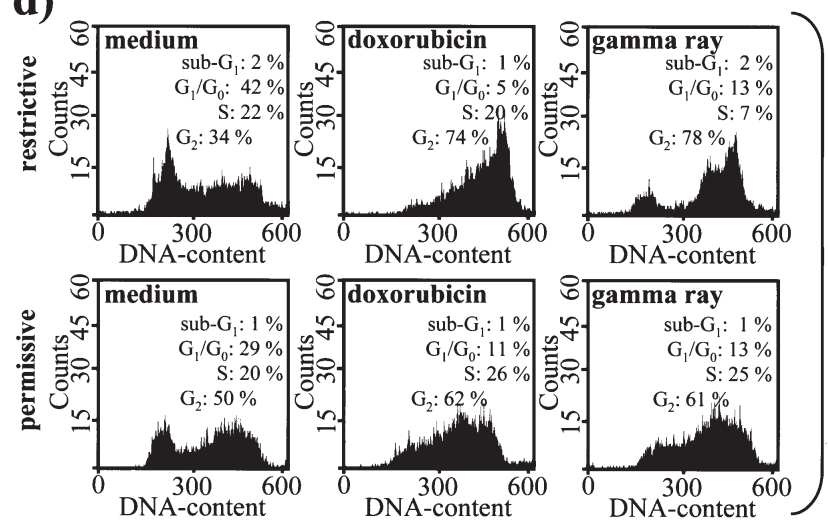

Figure 4. Flow cytometric analysis of cell cycle distribution after temperature shift, followed by treatment with doxorubicin, $\gamma$-radiation or medium. Cells were shifted to restrictive or permissive temperature for $24 \mathrm{~h}$, followed by the DNA damaging treatment $24 \mathrm{~h}$ before flow cytometry. (a) Cell cycle distribution of AsPC-1-cl.A cells. (b) Cell cycle distribution of AsPC-1-cl.B cells. (c) Cell cycle distribution of AsPC-1-v cells. (d) Cell cycle distribution of AsPC-1-pa cells. Histograms are representative of three independent experiments.

at permissive temperature was not noted in AsPC-1-pa and AsPC-1-v cells, and the doxorubicin- and radiation-induced $\mathrm{G}_{2}$-arrests were not abolished by the shift to permissive temperature. In summary, the cell cycle distribution of treated and non-treated AsPC-1 cells was similar only in cells expressing ts p53 at wt conformation (Fig. 4a and b). Apparently, wt p53 suppressed DNA damage-induced S-arrest (in the case of doxorubicin treatment) and $\mathrm{G}_{2}$-arrest (in the case of $\gamma$-irradiation) by the dominance of the p53-dependent $\mathrm{G}_{1}$-arrest.

\section{Discussion}

In the present study we introduced a novel cellular model to investigate the effect of restoration of wt p53 in pancreatic carcinoma cells. We retrovirally transduced a human temperature-sensitive p53 mutant into the p53-null pancreatic carcinoma cell line AsPC-1. In addition, we examined the effect of temperature-regulated wt p53 expression on DNA damaging treatment. A temperature shift to permissive temperature induced the $\mathrm{p} 53$ responsive gene $p 21^{\text {Wafl/Cipl }}$, indicative of proper ts $\mathrm{p} 53$ function. This observation is in line with the observed $G_{1}$ arrest at the permissive temperature. The level of expressed ts p53 decreased significantly following the shift to permissive temperature. This is explained by the conformational change of ts p53 into the wt p53 phenotype, which is more susceptible to Mdm2-dependent degradation (17). Interestingly, K-ras, which is constitutively active in AsPC-1 cells, is known to induce transcriptional upregulation of $\mathrm{Mdm} 2$ and subsequent p53 degradation $(17,18)$. In contrast to the induction of $p 21^{\text {Wafl/Cipl }}$, expression of the $\mathrm{p} 53$ responsive gene Bax remained unaltered following the shift to permissive temperature, and the cells did not undergo apoptosis. Previously, Zhao et al demonstrated that the transcriptional activation of $\mathrm{p} 53$ responsive genes depended on the p53 expression level. Limited expression of wt p53 was shown to suffice for $p 21^{\text {Wafl/Cipl }}$ induction, while transcriptional activation of proapoptotic genes, such as Pig3, required high levels of wt p53 (16). Hence, the down-regulation of ts p53 expression levels following the temperature shift might account for the lack of Bax upregulation at the permissive temperature. Notably, to the best of our knowledge, neither the Val-138 nor any other ts p53 mutant has ever been demonstrated to increase endogenous Bax protein expression in human tumor cells. Nevertheless, the ts Val-138 mutant has been shown to induce apoptosis in cell lines other than AsPC-1 (10).

The ability of p53 to induce its responsive genes also depends on posttranslational modifications, like Ser-46 phosphorylation, following exposure to DNA damaging drugs or $\gamma$-radiation $(15,19)$. Therefore, we subjected AsPC-1 ts p53 clones to doxorubicin treatment and $\gamma$-irradiation, both of 
which failed to induce Bax expression or apoptosis at the permissive temperature. At the restrictive temperature, however, these insults induced S- or $\mathrm{G}_{2}$-arrest. In contrast, AsPC-1 clones being $\mathrm{G}_{1}$-arrested as a result of wt p53 function completely lacked specific doxorubicin or radiation-induced alterations of cell cycle distribution. These results suggest that both mutant as well as wt p53 might influence the response of AsPC-1 cells to DNA damaging treatment in a disadvantageous way. On the one hand, the lack of functionally active p53 renders p53-dependent elimination of $\mathrm{G}_{2}$-arrested cells impossible. On the other hand, the presence of functionally active p53 prevents cells from progressing to S-phase of cell cycle, which is crucial for certain DNA damaging drugs and irradiation to execute their cytotoxicity. In support of this notion, previous studies have indicated that $\mathrm{p} 21^{\text {Waf } 1 / \text { Cip } 1}$-induced $\mathrm{G}_{1}$-arrest renders cancer cells resistant towards DNA damaging agents, such as doxorubicin (20). In summary, retroviral transduction of the human ts p53 mutant into AsPC-1 cells is a suitable model to investigate the function of wt $\mathrm{p} 53$ restoration in human pancreatic carcinoma cells.

\section{Acknowledgements}

We thank Drs K. Römer and N. Tsuchida for providing the ts p53 cDNA and S. Rathjen for excellent technical assistance. This study was supported by the 'Deutsche Krebshilfe' (Grant 10-1457).

\section{References}

1. Jemal A, Thomas A, Murray T and Thun M: Cancer statistics, 2002. CA Cancer J Clin 52: 23-47, 2002.

2. Hahn SA, Seymour AB, Shamsul Hoque ATM, Schutte M, Da Costa LT, Redston MS, Caldas C, Weinstein CL, Fischer A, Yeo CJ, Hruban RH and Kern SE: Allelotype of pancreatic adenocarcinoma using xenograft enrichment. Cancer Res 55: 4670-4675, 1995.

3. Moore S, Sipos B, Orlandini S, Sorio C, Real FX, Lemoine NR, Gress T, Bassi C, Klöppel G, Kalthoff H, Ungefroren H, Löhr M and Scarpa A: Genetic profile of 22 pancreatic carcinoma cell lines. Virchows Arch 439: 798-802, 2001.

4. Fridman JS and Lowe SW: Control of apoptosis by p53. Oncogene 22: 9030-9040, 2003.

5. Lundin J, Nordling S, von Boguslawsky K, Roberts PJ and Haglund C: Prognostic value of immunohistochemical expression of p53 in patients with pancreatic cancer. Oncology 53: 104-111, 1996.

6. Lane DP: P53, guardian of the genome. Nature 358: 15-16, 1992.

7. Berrozpe G, Schaeffer J, Peinado MA, Real FX and Perucho M: Comparative analysis of mutations in the p53 and K-ras genes in pancreatic cancer. Int J Cancer 58: 185-191, 1994.
8. Kimura M, Tagawa M, Takenaga K, Yamaguchi T, Saisho H, Nakagawara A and Sakiyama S: Inability to induce the alteration of tumorigenicity and chemosensitivity of p53-null human pancreatic carcinoma cells after the transduction of wild-type p53 gene. Anticancer Res 17: 879-884, 1997.

9. Lang D, Miknyoczki SJ, Huang L and Ruggeri BA: Stable reintroduction of wild-type p53 (MTmp53ts) causes the induction of apoptosis and neuroendocrine-like differentiation in human ductal pancreatic carcinoma cells. Oncogene 16: 1593-1602, 1998.

10. Yamato K, Yamamoto M, Hirano Y and Tsuchida N: A human temperature-sensitive p53 mutant p53Val-138: modulation of the cell cycle, viability and expression of p53-responsive genes. Oncogene 11: 1-6, 1995.

11. Chen WH, Horoszewicz JS, Leong SS, Shimano T, Penetrante R, Sanders WH, Berjian R, Douglass HO, Martin EW and Chu TM: Human pancreatic adenocarcinoma: in vitro and in vivo morphology of a new tumor line established from ascites. In Vitro 18: 24-34, 1982.

12. Chen WB, Lenschow W, Tiede K, Fischer JW, Kalthoff H and Ungefroren H: Smad4/DPC4-dependent regulation of biglycan gene expression by transforming growth factor- $\beta$ in pancreatic tumor cells. J Biol Chem 277: 36118-36128, 2002.

13. El-Deiry WS, Tokino T, Velculescu VE, Levy DB, Parsons R, Trent JM, Lin D, Mercer WE, Kinzler KW and Vogelstein B: WAF1, a potential mediator of p53 tumor suppression. Cell 75: $817-825,1993$.

14. Miyashita T and Reed JC: Tumor suppressor p53 is a direct transcriptional activator of the human bax gene. Cell 80: 293-299, 1995.

15. Oda K, Arakawa H, Tanaka T, Matsuda K, Tanikawa C, Mori T, Nishimori H, Tamai K, Tokino T, Nakamura Y and Taya Y: P53AIP1 a potential mediator of p53-dependent apoptosis, and its regulation by ser-46-phosphorylated p53. Cell 102: 849-862, 2000 .

16. Zhao R, Gish K, Murphy M, Yin Y, Notterman D, Hoffman WH, Tom E, Mack DH and Levine AJ: Analysis of p53-regulated gene expression patterns using oligonucleotide arrays. Genes Dev 14: 981-993, 2000.

17. Ries S, Biederer C, Woods D, Shifman O, Shirasawa S, Sasazuki T, McMahon M, Oren M and McCormick F: Opposing effects of Ras on p53: transcriptional activation of mdm2 and induction of p19ARF. Cell 103: 321-330, 2000

18. Cheng JQ, Ruggeri B, Klein WM, Sonoda G, Altomare DA, Watson DK and Testa JR: Amplification of AKT2 in human pancreatic cancer cells and inhibition of AKT2 expression and tumorigenicity by antisense RNA. Proc Natl Acad Sci USA 93: 3636-3641, 1996.

19. Rödicker F and Pützer BM: P73 is effective in p53-null pancreatic cancer cells resistant to wild-type TP53 gene replacement. Cancer Res 63: 2737-2741, 2003.

20. Mahyar-Roemer M and Roemer K: P21 Waf1/Cip1 can protect human colon carcinoma cells against p53-dependent and p53independent apoptosis induced by natural chemopreventive and therapeutic agents. Oncogene 20: 3387-3398, 2001. 